(2) Open Access Full Text Article

REVIEW

\title{
Exenatide extended-release; clinical trials, patient preference, and economic considerations
}

This article was published in the following Dove Press journal:

Patient Preference and Adherence

8 January 2013

Number of times this article has been viewed

\section{Sheila A Doggrell}

School of Biomedical Sciences, Faculty of Health, Queensland University of Technology, Brisbane, Australia
Correspondence: Sheila A Doggrell School of Biomedical Sciences, Gardens Point Campus, Faculty of Health, Queensland University of Technology, Brisbane, GPO 2343, QLD 400I, Australia

Email sheila.doggrell@qut.edu.au
Abstract: Type 2 diabetes remains an escalating problem worldwide, despite a range of treatments being available. The revelation that insulin secretion is under the control of a gut hormone, glucagon-like peptide 1, has led to a new paradigm in the management of type 2 diabetes, ie, use of medicines that directly stimulate or prolong the actions of endogenous glucagon-like peptide 1 at its receptors. Exenatide is an agonist at the glucagon-like peptide 1 receptor, and was initially developed as a subcutaneous medication twice daily (ExBID). Clinical trials with ExBID established a role for exenatide in the treatment of type 2 diabetes. Subsequently, exenatide once weekly (ExQW) was shown to have advantages over ExBID, and there is now more emphasis on the development of ExQW. ExQW alone reduces glycosylated hemoglobin $\left(\mathrm{HbA}_{1 \mathrm{c}}\right)$ and body weight, and is well tolerated. ExQW has been compared with sitagliptin, pioglitazone, and metformin, and been shown to have a greater ability to reduce $\mathrm{HbA}_{1 \mathrm{c}}$ than these other medicines. The only preparation of insulin with which ExQW has been compared is insulin glargine, and ExQW had some favorable properties in this comparison, notably causing weight loss compared with the weight gain on insulin glargine. ExQW has been compared with another glucagon-like peptide 1 receptor agonist, liraglutide, and was noninferior to liraglutide in reducing $\mathrm{HbA}_{1 \mathrm{c}}$. The small amount of evidence available shows that subjects with type 2 diabetes prefer ExQW to ExBID, and that adherence is high in the clinical trial setting. Health care and economic modeling suggests that ExQW will reduce diabetic complications and be cost-effective, compared with other medications, in long-term use. Little is known about whether subjects with type 2 diabetes prefer ExQW to other medicines, and whether adherence is good with ExQW in practice. These important topics require further study.

Keywords: exenatide, twice daily, once weekly, insulin, metformin, sitagliptin, type 2 diabetes

\section{Introduction}

The prevalence of diabetes in the US is about $8 \%$ (24 million people), and $90 \%$ is type 2 diabetes, which has both lifestyle and genetic components. ${ }^{1}$ Despite the treatments available for type 2 diabetes, about two thirds of subjects die from heart disease or stroke. Diabetes is also a leading cause of blindness, end-stage kidney failure, and lower limb amputations. ${ }^{1}$ Clearly, there is a need for good treatments for type 2 diabetes.

Medications are used in subjects with type 2 diabetes, when lifestyle changes have failed to manage their condition. Metformin is the medication of first choice in subjects with the disease. When lifestyle changes and the highest tolerated dose of metformin fail to provide control, a sulfonylurea is often added. The glitazones are an option as dual or additional therapy with metformin and/or a sulfonylurea. Finally, when oral 
medications do not control the disease, injectable insulin is added. Despite these medicines, type 2 diabetes is often progressive, with subjects unable to maintain long-term glycemic control. ${ }^{2}$ Also, some of these diabetic medicines (notably insulin and the sulfonylureas) increase body weight, which contributes further to diabetes.

Some recent physiological discoveries have opened up a new paradigm in the treatment of diabetes. In type 2 diabetes, hyperglycemia is due to a relative lack of insulin production and reduction in insulin sensitivity. ${ }^{1}$ Classically, glucose levels in the pancreatic cells, which relate to blood glucose levels, were considered to be the main controller of insulin levels. Recently, it has been discovered that gut peptides influence insulin secretion, and these peptides and their receptors are the target for a range of new drugs being introduced into the treatment of type 2 diabetes.

Glucagon-like peptide 1 (GLP-1) and gastric inhibitory peptide are released in response to a meal, and have already been reviewed in detail. ${ }^{3}$ Both of these gut hormones augment glucose-mediated insulin secretion, but only GLP-1 suppresses glucagon secretion, inhibits gastric emptying, and reduces appetite. ${ }^{3}$ Both GLP-1 and gastric inhibitory peptide are rapidly broken down by dipeptidyl peptidase-4, and thus have only short half-lives and biological effects. ${ }^{3}$ Production of both GLP-1 and gastric inhibitory peptide are severely reduced in subjects with type 2 diabetes. ${ }^{3}$ Levels of GLP-1 and gastric inhibitory peptide can be increased by inhibiting dipeptidyl peptidase-4, and this in turn will increase glucosemediated insulin secretion. ${ }^{3}$ The major difference between the sulfonylureas and drugs that increase the stimulation of the GLP-1 receptors is that the sulfonylureas increase insulin release independently of glucose, whereas stimulation of the GLP-1 receptor only augments glucose-mediated insulin secretion.

Exendin-4 is a 39-amino acid peptide, which acts as an agonist at GLP-1 receptors and is resistant to breakdown, as described previously. ${ }^{4}$ Synthetic exendin-4 (exenatide) is used to stimulate the GLP-1 receptor in type 2 diabetes. ${ }^{4}$ Exenatide augments glucose-mediated insulin secretion and inhibits glucagon secretion, and this decreases glycosylated hemoglobin $\left(\mathrm{HbA}_{1 \mathrm{c}}\right)$ and fasting plasma glucose levels. ${ }^{4}$ Exenatide also inhibits gastric emptying, and reduces appetite and food intake. ${ }^{4}$ Exenatide was initially used twice a day subcutaneously (ExBID) immediately before breakfast and dinner, but has recently been developed for once a week use (ExQW), and the subcutaneous injection does not need to be before a meal. Recently, several clinical trials and health care and economic modeling studies involving ExQW have been published, but there have been no reviews as yet that specifically relate to ExQW.

Initially, in this review, there is a brief outline of the clinical trials with ExBID, which established a role for exenatide in the treatment of type 2 diabetes. Subsequently, ExQW was shown to have advantages over ExBID, and there is now more emphasis on the development of ExQW. The comparison of ExQW with other antidiabetic drugs is discussed further on, and ExQW is shown to have some favorable properties compared with the other antidiabetic drugs. In describing clinical trials, only statistically significant differences are considered important, and when there is no significant difference, values are considered to be similar. ExQW is delivered subcutaneously, which contributes to patient preference, and this important issue is also discussed. To date, there are no clinical trials reporting clinical outcomes for ExQW, but the health care and economic considerations have been modeled, and this modeling is discussed. Finally, there is a commentary on the present findings, and the need for future research with ExQW.

\section{Exenatide twice daily}

ExBID has been shown to reduce $\mathrm{HbA}_{1 \mathrm{c}}$ and cause weight loss when used alone versus placebo, including in subjects with type 2 diabetes being treated with metformin, a sulfonylurea, or the combination of metformin and a sulfonylurea. ${ }^{4}$ ExBID has also been shown to decrease $\mathrm{HbA}_{1 \mathrm{c}}$ and body weight in subjects with type 2 diabetes being treated with a thiazolidinedione (pioglitazone or rosiglitazone) in the presence or absence of metformin. ${ }^{5}$ Recently, this has been extended to include in the presence of insulin. Thus, ExBID has been shown to reduce $\mathrm{HbA}_{1 \mathrm{c}}$ and body weight in subjects with poorly controlled type 2 diabetes, despite taking insulin glargine alone or in combination with metformin or pioglitazone (or both agents). ${ }^{6}$

Importantly, ExBID has been compared with the standard treatments for type 2 diabetes and shown to have some favorable effects. Two studies of subjects with inadequately controlled type 2 diabetes, despite using metformin and/or sulfonylurea therapy, randomized their subjects to either ExBID or insulin glargine, and showed that the reduction in $\mathrm{HbA}_{1 \mathrm{c}}$ was the same in both groups, but ExBID caused a reduction in body weight, whereas subjects taking insulin had an increase in body weight. However, ExBID caused more adverse gastrointestinal effects than insulin glargine in both studies. ${ }^{7,8}$ In another study, ExBID was compared with premixed insulin aspart in subjects with type 2 diabetes being treated with metformin, and shown to be noninferior 
to insulin in reducing $\mathrm{HbA}_{1 \mathrm{c}}$. Again, ExBID was shown to reduce body weight, whereas there was an increase with insulin aspart. ${ }^{9}$

When ExBID was compared with a sulfonylurea, glibenclamide, in subjects with uncontrolled type 2 diabetes despite receiving treatment with metformin, ExBID and glibenclamide decreased $\mathrm{HbA}_{1 \mathrm{c}}$ to a similar extent. With ExBID the subjects lost weight, whereas with glibenclamide they gained weight. Fasting insulin levels and insulin resistance were reduced by ExBID, but not by glibenclamide. ${ }^{10} \mathrm{~A}$ comparison of ExBID and another sulfonylurea (glimepiride) also showed that both agents caused a similar decrease in $\mathrm{HbA}_{1 \mathrm{c}}$, but ExBID was associated with body weight loss and reduced insulin resistance, whereas glimepiride did not have these effects. $^{11}$

An important finding regarding ExBID has come from a retrospective database analysis showing that it reduces the risk of cardiovascular events and hospitalizations. The data used were from the LifeLink database of medical and insurance claims. Subjects with type 2 diabetes but no history of cardiovascular events were assigned to an ExBID or non-exenatide cohort on their first new prescription filled. The cohorts were not matched at the start, because the 39,275 patients treated with ExBID were more likely to have ischemic heart disease, obesity, hyperlipidemia, hypertension, and/or other comorbidities at baseline than the 381,218 patients in the non-exenatide cohort. Nevertheless, going forward, the ExBID cohort had a smaller risk of cardiovascular disease events or hospitalizations than the non-exenatide group. ${ }^{12}$

ExBID has been associated with an increased incidence of acute pancreatitis during post-marketing surveillance. However, a retrospective cohort study of 786,656 subjects has shown an increased incidence of pancreatitis in diabetic versus nondiabetic subjects. The same study showed no association between ExBID and acute pancreatitis. ${ }^{13}$ Two other recent studies have confirmed that there is no relationship between ExBID and acute pancreatitis. ${ }^{14,15}$

\section{Efficacy, safety, and tolerability of ExQW versus ExBID ExQW alone}

The long-acting formulation of exenatide contains ExBID encapsulated in $0.06 \mathrm{~mm}$ diameter microspheres of medical grade poly-(D,L-lactide-co-glycolide). After mechanical suspension and subcutaneous injection, the microspheres hydrate in situ and adhere to one another to form an amalgam. Only the loosely bound surface exenatide (1\%) releases in the first few hours, whereas exenatide lodged in the deeper interstices diffuses out slowly, with a time to maximum concentration of about 2 weeks. Finally, fully encapsulated exenatide, which is inaccessible to diffusion, releases over a longer period, with a time to maximum of about 7 weeks, as already reviewed. ${ }^{16}$

In 62 subjects with type 2 diabetes, a single subcutaneous dose of ExQW at 2.5, 5, 7 or $10 \mathrm{mg}$ gave three peak plasma concentrations after about 3.5 hours, and 2 and 6 weeks, which represents the three phases of drug release from the microspheres. In 45 subjects with type 2 diabetes on weekly injections of ExQW at 0.8 and $2 \mathrm{mg}$, it was 2 weeks before ExQW $2 \mathrm{mg}$ reached the minimum effective concentration, which remained in the target therapeutic range until the injections were stopped. At the lower dose of ExQW, it took longer to reach the minimum effective concentration. Both doses of ExQW decreased $\mathrm{HbA}_{1 \mathrm{c}}$ and fasting plasma glucose, but only the higher dose of the ExQW ( $2 \mathrm{mg}$ ) reduced body weight. Nausea, which was mild, was observed in about one quarter of the subjects taking ExQW compared with 12\% in those on placebo. Although hypoglycemia was observed in $25 \%$ of subjects with type 2 diabetes on ExQW, none of it was severe. ${ }^{17}$

ExQW $2 \mathrm{mg}$ was tested in 43 subjects with type 2 diabetes and shown to be beneficial in comparison with placebo. The enrolled subjects had a mean $\mathrm{HbA}_{1 \mathrm{c}}$ of $8.5 \%$ and a fasting blood glucose of $9.9 \mathrm{mmol} / \mathrm{L}$, and most $(\mathrm{n}=27)$ were receiving metformin. The subjects were randomized to placebo or ExQW at 0.8 or $2 \mathrm{mg}$ weekly, and those on the $2 \mathrm{mg}$ dose reached a therapeutic concentration $(50 \mathrm{pg} / \mathrm{mL})$ within 2 weeks and retained it for 15 weeks, whereas those on the $0.8 \mathrm{mg}$ only reached a borderline therapeutic concentration. When the ExQW was stopped after week 15, it was 3 weeks before the plasma concentrations became too low to be therapeutic. After 15 weeks, $\mathrm{HbA}_{1 \mathrm{c}}$ was reduced by $1.8 \%$ and $2.1 \%$ on ExQW 0.8 and $2 \mathrm{mg}$, respectively, and fasting plasma glucose was reduced by about $2.4 \mathrm{mmol} / \mathrm{L}$ on each dose strength. The lower dose of exenatide had no effect on body weight whereas exenatide $2 \mathrm{mg}$ caused a weight loss of $3.8 \mathrm{~kg}$. The most common adverse effect was nausea, followed by gastroenteritis and bruising at the injection site. There was no major hypoglycemia with ExQW over 15 weeks. $^{18}$

In 29 Japanese subjects with type 2 diabetes suboptimally controlled on metformin, a sulfonylurea, a thiazolidinedione, or a combination of these, exenatide $0.8 \mathrm{mg}$ and $2 \mathrm{mg}$ once weekly gave steady-state concentrations by week 8 . At 10 weeks, exenatide 0.8 and $2 \mathrm{mg}$ once weekly had reduced 
$\mathrm{HbA}_{1 \mathrm{c}}$ by $0.6 \%$ and $1.1 \%$, respectively. Only $2 \mathrm{mg}$ of exenatide had a definite ability to reduce fasting and postprandial glucose. The most common side effect was mild to moderate hardening/thickening of the skin at the injection site followed by mild nausea, and no serious hypoglycemia was observed. Treatment-emergent antibodies to exenatide were present in about two thirds of treated subjects, but did not affect changes in $\mathrm{HbA}_{1 \mathrm{c}}{ }^{19}$

\section{ExQW compared with ExBID}

ExBID and ExQW have been compared in the DURATION-1 (Diabetes Therapy Utilization: Changes in $\mathrm{A}_{1 \mathrm{c}}$, Weight, and Other Factors Through Intervention with Exenatide Once Weekly) study, and the once-weekly formulation was shown to be at least as good as the twice-daily formulation. DURATION-1 was an open-label, noninferiority comparison between ExBID and ExQW. The DURATION-1 study enrolled 295 subjects with type 2 diabetes, a mean age of 55 years, a baseline $\mathrm{HbA}_{1 \mathrm{c}}$ of $8.3 \%$, and fasting plasma glucose of about $8.4 \mathrm{mmol} / \mathrm{L}$, who were being managed by diet and exercise (approximately 15\%), metformin alone (about $36 \%$ ), or in combination with other drugs (about 38\%), or other antidiabetic drugs. Subjects were randomized, and then underwent a 3-day lead-in period of exenatide $5 \mu \mathrm{g}$ twice daily, before receiving exenatide $2 \mathrm{mg}$ once a week or exenatide $5 \mu \mathrm{g}$ twice daily for 28 days followed by $10 \mu \mathrm{g}$ twice daily for 30 weeks. It only took 2 weeks to obtain the therapeutic range of exenatide with the once-weekly preparation. The two preparations had similar effects on $\mathrm{HbA}_{1 \mathrm{c}}$ over 9 weeks, beyond which ExQW caused a greater reduction in $\mathrm{HbA}_{1 \mathrm{c}}(1.9 \%$ at 30 weeks versus $1.5 \%$ with ExBID). ExQW also caused a greater reduction in fasting plasma glucose $(2.3 \mathrm{mmol} / \mathrm{L})$ than ExBID $(1.4 \mathrm{mmol} / \mathrm{L})$, whereas ExBID caused a greater reduction in postprandial glucose $(6.9 \mathrm{mmol} / \mathrm{L})$ than ExQW $(5.3 \mathrm{~mol} / \mathrm{L})$. Both preparations of exenatide caused a similar decrease in low-density lipoprotein cholesterol, triglycerides, systolic and diastolic blood pressure, and body weight (about $3.65 \mathrm{~kg}$ ). Nausea was more common with ExBID than with ExQW (35\% versus 26\%), as was vomiting (19\% versus $11 \%)$, upper respiratory tract infection (17\% versus $8 \%$ ), and bruising at the injection site ( $10 \%$ versus $5 \%$ ). The incidence of diarrhea, constipation, and urinary tract infection was similar with both preparations, but pruritus at the injection site was more common with ExQW $(18 \%)$ than with ExBID (1\%). ${ }^{20}$

DURATION-1 has been followed up at 52 weeks, with subjects taking ExQW continuing on this therapy, and the subjects with type 2 diabetes taking ExBID being switched to ExQW $2 \mathrm{mg}$. In the subjects continuing with ExQW, the reduction in $\mathrm{HbA}_{1 \mathrm{c}}$ was maintained at 52 weeks, whereas those who switched to ExQW had an improvement in $\mathrm{HbA}_{1 \mathrm{c}}$. Subjects on ExBID who were switched to ExQW had a transient increase in fasting blood glucose, followed by a decrease after 2 weeks, and this increase was probably due to short-term subtherapeutic levels of exenatide. By 52 weeks, the reduction in diastolic and systolic blood pressure on both exenatide protocols had reached clinical significance, with decreases of 6.2/2.8 $\mathrm{mmHg}$ in those continuing on ExQW. The switch to ExQW was associated with an increase in pruritus at the injection site. There were no episodes of major hypoglycemia, and only minor hypoglycemia occurred in subjects taking sulfonylureas. ${ }^{21}$

A further follow-up of DURATION-1 out to 2 years showed that ExQW continued to be well tolerated. Follow-up also demonstrated sustained glucose control, weight loss, and blood pressure reductions. There were also small decreases in cholesterol, low-density lipoprotein cholesterol, and triglycerides. $^{22}$

DURATION-5 was similar in design to DURATION-1, and had similar findings. DURATION-5 enrolled 252 subjects with type 2 diabetes and an $\mathrm{HbA}_{1 \mathrm{c}}$ of $8.4 \%$, and the reduction in $\mathrm{HbA}_{1 \mathrm{c}}$ after 24 weeks was greater with ExQW (1.6\%) than with ExBID (0.9\%). Weight loss was similar in both groups. Heart rate increased by 4.1 and 2.1 beats per minute with ExQW and ExBID, respectively. Nausea was less common with ExQW than with ExBID (14\% versus $35 \%$ ), as was vomiting ( $5 \%$ versus $9 \%$ ), but erythema at the injection site was more common with ExQW than with ExBID (5.4\% versus 2.4\%). ${ }^{23}$

The results from DURATION-1 and DURATION-5 have been combined for analysis of safety and tolerability. Nausea was less common with ExQW (20.9\%) than with ExBID (35.7\%), as was vomiting (7.9\% versus $14.2 \%$, respectively), and both of these adverse effects decreased in incidence with time. Erythema and pruritus at the injection site were more common with ExQW than ExBID (erythema, 6.5\% versus $1.1 \%$; pruritus, $11.9 \%$ versus $1.1 \%$ ), and these adverse effects also decreased over time. No subjects had major hypoglycemia, but subjects in both groups using sulfonylureas did have some minor hypoglycemia. ${ }^{24}$

Subpopulation analysis of the data with ExQW has been undertaken by Amylin Pharmaceuticals (San Diego, CA), the company developing ExQW. This analysis included data from DURATION 1-6, and another trial that has not been published as yet in a peer-reviewed journal. ExQW reduced $\mathrm{HbA}_{1 \mathrm{c}}$ levels, fasting glucose, and body weight in 
all subpopulations ( $<$ or $\geq 65$ years, gender, race, duration of diabetes, and body mass index). Low-density lipoprotein, cholesterol, and blood pressure was reduced in all subpopulations except Blacks. Hypoglycemia was uncommon (2.3\%) except in subjects who were also taking sulfonylureas. ${ }^{25}$

\section{Comparative clinical trials of ExQW and other antidiabetic drugs Comparison with sitagliptin, pioglitazone, and metformin}

ExQW has been compared with sitagliptin, pioglitazone, and metformin and shown to have a favorable profile compared with each of these agents. In DURATION-2, ExQW was compared with sitagliptin or pioglitazone in subjects with type 2 diabetes taking metformin in a double-blind, double-dummy clinical trial. The 491 enrolled subjects had a mean age of 52 years and an $\mathrm{HbA}_{1 \mathrm{c}}$ of about $8.5 \%$, and were randomized to ExQW $2 \mathrm{mg}$, sitagliptin $100 \mathrm{mg}$, or pioglitazone $45 \mathrm{mg}$ for 26 weeks. The $\mathrm{HbA}_{1 \mathrm{c}}$ levels were lowered to $7.2 \%$ with ExQW, which was significantly greater than the $7.7 \%$ with sitagliptin or $7.4 \%$ with pioglitazone. Fasting blood glucose was lowered by $1.8 \mathrm{mmol} / \mathrm{L}$ with ExQW, which was similar to that with pioglitazone $(1.5 \mathrm{mmol} / \mathrm{L})$ but greater than with sitagliptin (0.9 mmol/L). Subjects taking ExQW lost $2.3 \mathrm{~kg}$, which was greater than the loss with sitagliptin $(0.8 \mathrm{~kg})$, whereas subjects taking pioglitazone gained $2.8 \mathrm{~kg}$. ExQW caused a reduction in systolic blood pressure $(4 \mathrm{mmHg})$, whereas sitagliptin and pioglitazone had little effect on blood pressure after 16 weeks. $^{26}$

There was no major hypoglycemia with ExQW, sitagliptin, or pioglitazone in DURATION-2. Minor hypoglycemia occurred in $1 \%$ of subjects with type 2 diabetes taking ExQW, $3 \%$ of those on sitagliptin, and $1 \%$ of those on pioglitazone. The most common adverse effects were nausea and diarrhea with ExQW and sitagliptin. With pioglitazone, the most common adverse effects were upper respiratory tract infection and peripheral oedema. ${ }^{26}$

Subsequently, all subjects with type 2 diabetes in DURATION-2 received ExQW. After 26 weeks, the subjects who changed from sitagliptin to ExQW had reduced $\mathrm{HbA}_{1 \mathrm{c}}$, fasting plasma glucose, and weight, whereas subjects who were changed from pioglitazone to ExQW has no change in $\mathrm{HbA}_{1 \mathrm{c}}$ or fasting blood glucose, but did lose weight. ${ }^{27}$

The DURATION-4 trial compared ExQW with metformin $2 \mathrm{~g} /$ day, sitagliptin $100 \mathrm{mg} /$ day, or pioglitazone $45 \mathrm{mg}$ /day in 822 subjects with type 2 diabetes who were diabetes drug-naïve. After 26 weeks, $\mathrm{HbA}_{1 \mathrm{c}}$ was decreased by
$1.5 \%$ by ExQW, $1.5 \%$ by metformin, $1.6 \%$ by pioglitazone, and $1.2 \%$ by sitagliptin. Body weight decreased with ExQW, metformin, and sitagliptin, but increased with pioglitazone. Nausea and injection site reactions were only observed with ExQW. There were no incidents of major hypoglycemia. ${ }^{28}$

\section{Comparison with insulin}

The only preparation of insulin which ExQW has been compared with in a peer-reviewed journal is insulin glargine, and ExQW showed some favorable properties in this comparison. In DURATION-3, ExQW was compared with insulin glargine in subjects with type 2 diabetes. In this open-label, randomized, parallel-group Phase III study, 456 subjects with suboptimal glycemic control despite maximum tolerated doses of metformin or a combination of metformin and sulfonylureas were randomized to ExQW or insulin glargine. Subjects started insulin glargine 10 IU/day, measured their fasting blood glucose concentrations every morning, and adjusted insulin doses to achieve a plasma glucose of 4.0-5.5 mmol/L. After 26 weeks, the reduction in $\mathrm{HbA}_{1 \mathrm{c}}$ from a baseline of $8.3 \%$ was $1.5 \%$ with ExQW, which was significantly greater than the $1.3 \%$ with insulin glargine. There was a larger reduction in fasting blood glucose with insulin glargine $(2.8 \mathrm{mmol} / \mathrm{L})$ than with ExQW $(2.1 \mathrm{mmol} / \mathrm{L})$, but a bigger reduction in postprandial glucose with ExQW than insulin glargine. Body weight decreased by $2.6 \mathrm{~kg}$ with ExQW, but increased by $1.4 \mathrm{~kg}$ with insulin glargine. ExQW reduced blood pressure by $4.2 / 1.5 \mathrm{mmHg}$ and increased heart rate by 1.97 beats per minute, whereas insulin glargine only reduced systolic blood pressure and had no effect on heart rate. ${ }^{29}$

In DURATION-3, minor hypoglycemia was observed in fewer subjects receiving ExQW (8\%) than those on insulin glargine $(26 \%)$, as was the occurrence of symptoms of hypoglycemia ( $13 \%$ versus $31 \%$ ). Gastrointestinal side effects were more common with ExQW than insulin glargine, whereas nasopharyngitis and headache were more common with insulin than exenatide. One subject taking ExQW had edematous pancreatitis but made a full recovery. $^{29}$

ExQW has also been compared with insulin glargine in 427 Japanese subjects with type 2 diabetes and already taking oral drugs for treatment of diabetes. After 26 weeks, $\mathrm{HbA}_{1 \mathrm{c}}$ was reduced by $1.1 \%$ and $0.7 \%$ in the ExQW and insulin groups, respectively. Body weight was decreased by ExQW but increased slightly with insulin glargine. ExQW decreased low-density lipoprotein cholesterol, whereas insulin did not. ExQW had a lower risk of hypoglycemia 
than insulin glargine, but a higher risk of adverse effects at the injection site. ${ }^{30}$

\section{Comparison with liraglutide}

ExQW was compared with liraglutide in DURATION-6. Liraglutide is also an agonist at the GLP-1 receptor, and is used subcutaneously at $1.8 \mathrm{mg}$ once a day in the treatment of type 2 diabetes. DURATION-6 enrolled 911 subjects with type 2 diabetes, which was poorly controlled despite the use of metformin, a sulfonylurea, or metformin and a sulfonylurea or pioglitazone. In the first four weeks of the trial, 15 subjects in the liraglutide group withdrew because of adverse gastrointestinal effects, whereas none in the ExQW withdrew for this reason. At baseline, $\mathrm{HbA}_{1 \mathrm{c}}$ was about $8.4 \%$, and after 26 weeks was reduced by $1.3 \%$ on ExQW and $1.5 \%$ on liraglutide. Although the effect on $\mathrm{HbA}_{1 \mathrm{c}}$ was greater with liraglutide than with ExQW, statistical analysis showed that ExQW was noninferior to liraglutide. Weight loss was greater with liraglutide than with ExQW (about $3.5 \mathrm{~kg}$ versus $2.8 \mathrm{~kg}$ at 26 weeks). Adverse gastrointestinal effects remained more common with liraglutide than with ExQW (nausea, 21\% versus 9\%; vomiting, 11\% versus $4 \%$; diarrhea, $13 \%$ versus $6 \%$ ), whereas injection site nodules were more common with ExQW than with liraglutide ( $3 \%$ versus $<1 \%$ ). No major hypoglycemia was observed with either drug, but minor hypoglycemia in the absence of a sulfonylurea was observed in 3\% of subjects taking liraglutide and 4\% taking ExQW. In subjects taking sulfonylureas, the rate of minor hypoglycemia was $12 \%$ with liraglutide and $15 \%$ with ExQW. ${ }^{31}$

A meta-analysis that combined all the information for ExQW and liraglutide showed that $\mathrm{HbA}_{1 \mathrm{c}}$ was lowered by $1.15 \%$ on ExQW, $1.01 \%$ on liraglutide $1.2 \mathrm{mg}$, and $1.18 \%$ on liraglutide $1.8 \mathrm{mg}$, and concluded that there were no meaningful differences in $\mathrm{HbA}_{1 \mathrm{c}}$-lowering with ExQW and the two doses of liraglutide. ${ }^{32}$

\section{Patient preference and adherence}

In DURATION-1, treatment adherence (measured as injections received/injections planned) was $98 \%$ for both ExBID and ExQW over 30 weeks. ${ }^{19}$ During DURATION-1 and its extension to 52 weeks, treatment satisfaction was assessed using the Diabetes Treatment Satisfaction Questionnaire (DTSQ). ${ }^{33}$ The DTSQ assesses six items, ie, "current treatment", "convenience", "flexibility", "understanding", "recommend", and "continue", and uses a Likert scale of 0-7 for each item. After 30 weeks of ExQW, there were improvements for all items except for perceived hypoglycemic frequency.
After 30 weeks, subjects taking ExBID were switched to ExQW, and this improved the DTSQ score. The Impact of Weight on Quality of Life Questionnaire-Lite (IWQOL), ${ }^{34}$ which has domains on "physical function", "self-esteem", "sex life", "public distress", and "work" was improved by both ExQW and ExBID. Furthermore, when subjects switched from ExBID to ExQW, there was a further improvement in quality of life. Nausea with exenatide did not alter the improvements in DTSQ score or IWQOL. ${ }^{35}$

In DURATION-2, ExQW was more effective than sitagliptin or pioglitazone in reducing $\mathrm{HbA}_{1 \mathrm{c}}$, and also reduced body weight. ${ }^{26} \mathrm{After} 26$ weeks, the DTSQ score was improved by ExQW, sitagliptin, and pioglitazone in DURATION-2. However, the improvement was greater with ExQW than with sitagliptin. IWQOL was improved by ExQW and sitagliptin, but not by pioglitazone, which increased body weight. The Psychological General Well-Being (PGWB) index has six dimensions, ie, anxiety, depressed mood, positive well-being, self-control, general health, and vitality. ${ }^{36} \mathrm{ExQW}$, sitagliptin, and pioglitazone all improved the PGWB. ${ }^{26,37}$

There is very little information about adherence with exenatide. In DURATION-1, adherence with ExQW and ExBID was very high at $98 \%$ for both formulations over 30 weeks. ${ }^{20}$ A retrospective cohort analysis of adherence with ExBID or insulin glargine used the HealthCore Integrated Research Database ${ }^{\mathrm{TM}}$, and calculated adherence as the 12-month medication ratio, ie, the aggregate days of supply of all fills for therapy observed over 12 months after initiation divided by 365 days. Adherence was $68 \%$ with ExBID, which was significantly higher than the $58 \%$ for insulin glargine..$^{38}$

\section{Health care and economic considerations Center for Outcomes Research model}

The Center for Outcomes Research (CORE) has developed a diabetes model which determines the long-term health outcomes and economic consequences of implementing different treatment strategies. The model takes account of baseline cohort characteristics, any past history of complications, current and future diabetes management, and concomitant medications. From this model, development of complications, life expectancy, quality-adjusted life expectancy, and total costs within populations can be calculated. ${ }^{39}$

In subjects with poorly controlled diabetes, despite use of metformin and/or sulfonylureas, Phase III clinical trials have shown that ExBID reduced $\mathrm{HbA}_{1 \mathrm{c}}{ }^{40-42}$ and these data have been used in the CORE diabetes model to determine 
the effect of adding ExBID versus not adding ExBID. This modeling showed that the increased cost over 30 years with ExBID was associated with increased life expectancy and increased quality-adjusted life expectancy. ${ }^{43}$

CORE has also been applied to DURATION-2, in which ExQW was compared with sitagliptin or pioglitazone in subjects with poorly controlled diabetes, despite taking metformin, and it was shown that subjects on ExQW achieved a bigger reduction in $\mathrm{HbA}_{1 \mathrm{c}}$ than those on sitagliptin or pioglitazone. ${ }^{26}$ In this model, use of ExQW was extended beyond the 6 months of DURATION-2, with a deterioration of glycemic control until the subjects were switched to basal insulin after 3 years. After 35 years, in the CORE modeling of DURATION-2, ExQW increased life expectancy by 0.28 and 0.17 years, and quality-adjusted life years by 0.28 and 0.24 years, compared with sitagliptin and pioglitazone, respectively. The risk of 14 of 16 diabetic complications (eg, myocardial infarction, renal disease, eye disease, ulcer, neuropathy) was lower with ExQW than with sitagliptin, but for two other complications, ie, first stroke and heart failure, the risk was slightly higher with ExQW than with sitagliptin. The relative risk of 13 of 16 complications was also lower with ExQW than with pioglitazone, but the risk of acute myocardial infarction, stroke, and angina was slightly higher with ExQW than with pioglitazone. ExQW had lower complication costs of US\$2215 and US\$933 versus sitagliptin and pioglitazone, respectively. The biggest limitation in this study is that a relative short clinical trial of 6 months with surrogate endpoints only had been used in the modeling over 35 years. ${ }^{44}$

The CORE model has also been applied to DURATION-3, the trial showing that subjects with suboptimally managed diabetes despite taking metformin alone or with sulfonylureas had a bigger reduction in $\mathrm{HbA}_{1 \mathrm{c}}$ with ExQW than with insulin glargine $10 \mathrm{IU} /$ day, and weight loss was observed with ExQW, whereas insulin glargine increased body weight. ${ }^{29}$ From the CORE diabetes model, life expectancy and quality-adjusted life expectancy were higher with ExQW than with insulin glargine, and there was also an increased time to diabetic complications with ExQW compared with insulin glargine. At the time of the analysis, the cost of ExQW was unknown, but if it was the same as liraglutide $1.8 \mathrm{mg}$, the ExQW would be more costly than insulin glargine. However, it should be noted that this was partly due to the increased life expectancy with ExQW, and thus may be acceptable. ${ }^{45}$

Another group applied the CORE diabetes model to DURATION-3, with the known price of ExQW in the US, and came up with similar results. Compared with insulin glargine, ExQW over 35 years would reduce the risk of cardiovascular disease (except stroke), renal disease, eye disease, and ulcer/neuropathy. ExQW would increase life expectancy and quality-adjusted life expectancy, and this would lead to increased costs with ExQW compared with insulin glargine of US\$15,936 per quality-adjusted life year, which is under the acceptable rate for a quality-adjusted life year of US\$50,000. ${ }^{46}$

The LEAD-6 (Liraglutide Once Daily Compared With Exenatide Twice Daily) trial comparing liraglutide with ExBID was also subjected to the CORE diabetes model, which showed that liraglutide improved quality of life and reduced the incidence of diabetes-related complications compared with ExBID. Liraglutide was cost-effective from a health care perspective in Switzerland, Denmark, Norway, The Netherlands, and Austria. ${ }^{47}$ As noted in the previous section, ExQW is more effective than ExBID at lowering $\mathrm{HbA}_{1 \mathrm{c}}$ and fasting glucose, and is more convenient to use than ExBID. Thus, a more appropriate comparison would be liraglutide and ExQW.

\section{The Archimedes model}

Another model/simulation has been used to predict cardiovascular outcomes with a once-weekly GLP-1 receptor agonist such as ExQW in subjects with type 2 diabetes. The Archimedes model is a clinically detailed simulation model of human physiology, disease progression, and health care delivery which was initially developed as a diabetes model. ${ }^{48}$ The Archimedes diabetes model was then validated against more than 50 major clinical trials, including some in subjects with diabetes. ${ }^{49}$

For the Archimedes diabetes modeling of the effect of GLP-1 agonists, the population used in the simulation study were derived from the National Health and Nutrition Examination Survey (NHANES). In the NHANES virtual population with type 2 diabetes, the $\mathrm{HbA}_{1 \mathrm{c}}$ level is $8.1 \%$ despite use of metformin with or without a sulfonylurea. ${ }^{50}$ Four populations were simulated, ie, standard care, intensive glycemic control, glycemic control and weight reduction, and one with additional improvements in systolic blood pressure and lipids, with these last two simulations representing the effects of GLP-1 receptor agonist. In this model, the GLP-1 receptor agonist achieved a decrease in $\mathrm{HbA}_{1 \mathrm{c}}$ over the first year, with some upward drift over time. By year 20, intensive glycemic control led to a $6.1 \%$ reduction in macrovascular events relative to standard care, and this represented a reduction in fatal and nonfatal myocardial infarction, stroke, and 
death from coronary artery disease of $9.2 \%, 2.2 \%$, and $10.2 \%$, respectively. With glycemic control and weight reduction, the rates of myocardial infarction, stroke, and coronary death were increased to $19.5 \%, 6.2 \%$, and $17.6 \%$, respectively. With the addition of reduced blood pressure and lipid controls, the reductions in myocardial infarction, stroke, and coronary death were $26.7 \%, 10.7 \%$, and $24.8 \%$, respectively. Only the GLP-1 receptor agonist simulations led to a reduction in the early signs of renal disease (macroalbuminuria). ${ }^{51}$

The Archimedes model and the NHANES population have also been used to compare ExQW with insulin and pioglitazone in subjects with type 2 diabetes taking metformin. The model included both moderate and high adherence with insulin. Data from four of the DURATION trials $(1,2,3$, and 5) were used to quantify the effects of ExQW. With this model, after one year, $\mathrm{HbA}_{1 \mathrm{c}}$ decreased by about $1.5 \%$ with ExQW, which was more than the $1 \%$ with pioglitazone and high adherence with insulin and the $0.5 \%$ with moderate adherence with insulin. Body weight decreased with ExQW, but increased with insulin and pioglitazone. After 20 years, major adverse cardiovascular events were lower in this simulation with ExQW than with high or moderate adherence to insulin or pioglitazone. At 5 years, there were increased life years and quality-adjusted life years with ExQW, compared with insulin and pioglitazone, and this continued up to the 20-year mark. A limitation of this study was that subjects with type 2 diabetes were assumed to be $100 \%$ adherent with ExQW, with high adherence to insulin and pioglitazone, which is not realistic. ${ }^{52}$

\section{Commentary}

\section{Long-term studies of clinical endpoints and safety with ExQW}

The trials of ExQW discussed in this review have described the effects on surrogate endpoints (eg, $\mathrm{HbA}_{1 \mathrm{c}}$ ) for type 2 diabetes. The much more important endpoints are clinical ones, such as myocardial infarction and hospitalization. Recently, a retrospective cohort trial has suggested that ExBID is associated with a lower risk of cardiovascular disease events and hospitalizations than treatment with other glucose-lowering therapies, ${ }^{12}$ but such information is not available from a prospective trial or for ExQW. The modeling studies suggest that ExQW will reduce cardiovascular endpoints, but it is important that clinical trials or evaluations are undertaken to determine whether ExQW does lower the incidence of cardiovascular disease and hospitalizations.

Based on postmarketing data, ExBID was associated with acute pancreatitis, including fatal and nonfatal hemorrhagic or necrotizing pancreatitis. ${ }^{53}$ Although recent evidence suggests that ExBID is not linked with pancreatitis, as discussed earlier, it does raise the issue of long-term safety with ExQW, which is yet to be evaluated in large numbers of subjects with type 2 diabetes.

\section{Gliptins}

The gliptins inhibit dipeptidyl peptidase-4 and increase levels of endogenous glucagon-like peptide-1 to augment glucosemediated insulin secretion. To date, ExQW has only been compared with one dipeptidyl peptidase-4 inhibitor, which was sitagliptin, and ExQW was shown to lower $\mathrm{HbA}_{1 \mathrm{c}}$ to a greater extent than sitagliptin. It has been suggested that vildagliptin is more potent than sitagliptin at reducing $\mathrm{HbA}_{1 \mathrm{c}}$ in Japanese subjects with type 2 diabetes. ${ }^{54}$ Thus, it is possible that ExQW may not be more potent than gliptins other than sitagliptin. Thus, a direct comparison of ExQW and vildagliptin should be undertaken in subjects with type 2 diabetes. Further, it is possible that the effects of ExQW and the gliptins are additive, with ExQW directly stimulating the receptor for glucagon-like peptide-1, and the gliptins causing a buildup of endogenous glucagon-like peptide-1, which also stimulates the receptor. This should be tested in subjects with poorly managed type 2 diabetes despite using either ExQW or a gliptin.

\section{Comparison with sulfonylureas}

When metformin is ineffective at controlling type 2 diabetes alone, the standard group of drugs added are the sulfonylureas. Although effective at reducing $\mathrm{HbA}_{1 \mathrm{c}}$ in the presence of metformin, the sulfonylureas increase the incidence of hypoglycemia. Thus, when type 2 diabetes is not controlled by metformin alone, ExQW should be trialed as an alternative to a sulfonylurea. Such a trial should compare not only the incidence of hypoglycemia between ExQW and a sulfonylurea, but also efficacy and other aspects of safety in subjects with type 2 diabetes being poorly managed with metformin.

\section{Add-on to insulin}

Adding ExBID to insulin glargine has been shown to be effective in the treatment of subjects with type 2 diabetes. Thus, addition of ExBID is associated with a lowering of $\mathrm{HbA}_{1 \mathrm{c}}$, lower basal insulin requirements, and with weight loss or less weight gain, as extensively reviewed elsewhere. ${ }^{55}$ However, the effects of adding ExQW to insulin glargine have not been assessed in a clinical trial, and should be. 


\section{Preference and adherence}

There is limited information available for preference and adherence with ExQW. ExQW was preferred to ExBID in DURATION-1, as discussed earlier. However, it is not known whether ExQW is preferred to other diabetic medicines, and this needs to be investigated.

Because it takes 3 weeks to drop from steady state to below therapeutic levels with ExQW, ${ }^{18}$ subjects with type 2 diabetes who miss the occasional dose of ExQW may maintain reasonable glycemic control, and this needs to be investigated. Other antidiabetic medicines, including ExBID, have shorter durations of action than ExQW, and nonadherence with these other diabetic medicines may be associated with poorer glycemic control than with ExQW. This comparison also needs to be undertaken. Very high adherence to ExQW has been reported in the clinical trial setting,,$^{20}$ but this needs to be assessed in practice. Higher adherence with ExBID than with insulin glargine has been reported, ${ }^{35}$ but comparison of adherence rates between ExQW and insulin glargine needs investigating.

\section{Archimedes diabetes simulation and AHEAD trial}

In the modeling of a GLP-1 agonist in diabetes, Peskin et al considered the agonist to be equivalent to glycemic control and weight reduction or these plus additional improvements in systolic blood pressure and lipids. ${ }^{51}$ This modeling suggested that there would be improvements in cardiovascular outcomes associated with these changes. ${ }^{51}$ The Action for Health in Diabetes (Look AHEAD) trial was designed to determine whether intensive lifestyle changes compared with diabetes support and education would improve cardiovascular outcomes. ${ }^{56}$ In this trial, after 4 years, physical activity was greater, weight loss was about $5 \mathrm{~kg}$, and $\mathrm{HbA}_{1 \mathrm{c}}$ was lowered by $0.5 \%$ in the lifestyle group. ${ }^{57}$ However, there was no evidence of improved cardiovascular outcomes in the intensive lifestyle change group, compared with the diabetes support and education group, and the trial has been abandoned for futility. ${ }^{58}$

Given that Archimedes modeling of the GLP-1 agonist was based on it being equivalent to lifestyle changes, ${ }^{51}$ similar results from the simulation and real trial might be expected, but this did not occur. One possible explanation for this is that the modeling produced much larger decreases in body weight and $\mathrm{HbA}_{1 \mathrm{c}}$ than were observed in the Look AHEAD trial, and these larger decreases are needed to achieve an improvement in cardiovascular outcomes.

\section{Conclusion}

The clinical trials with ExBID established a role for exenatide in the treatment of type 2 diabetes. Subsequently, ExQW was shown to have advantages over ExBID, and there is now more emphasis on the development of ExQW. ExQW alone reduces $\mathrm{HbA}_{1 \mathrm{c}}$ and body weight, and is well tolerated. ExQW has been compared with sitagliptin, pioglitazone, and metformin, and shown to have a greater ability to reduce $\mathrm{HbA}_{1 \mathrm{c}}$ than these other medicines. ExQW has been compared with insulin glargine, and ExQW causes weight loss compared with a weight gain with insulin glargine. ExQW has been compared with another GLP-1 receptor agonist, liraglutide, and the results suggest that ExQW is noninferior to liraglutide. The small amount of evidence available shows that subjects with type 2 diabetes prefer ExQW to ExBID, and that adherence was high in the clinical trial setting. Health care and economic modeling suggests that ExQW will reduce diabetic complications and be costeffective compared with other medications in long-term use. Little is known about whether subjects with type 2 diabetes prefer ExQW to other medicines and whether adherence with ExQW is good in practice, and these important topics require further study.

\section{Disclosure}

The author reports no conflicts of interest in this work.

\section{References}

1. Ligaray KPL, Isley WL. Diabetes mellitus, type 2. Available from: http://emedicine.medscape.com/article/117853-overview. Accessed January 26, 2010

2. Koro CE, Bowlin SJ, Bourgeois N, Fedder DO. Glycemic control from 1988 to 2000 among US adults diagnosed with type 2 diabetes: a preliminary report. Diabetes Care. 2004;27:17-20.

3. Zarrinpar A, Loomba R. Review article: the emerging interplay among the gastrointestinal tract, bile acids and incretins in the pathogenesis of diabetes and non-alcoholic fatty liver disease. Aliment Pharmacol Ther. 2012;36:909-921

4. Doggrell SA. Is exenatide improving the treatment of type 2 diabetes? Analysis of the individual clinical trials with exenatide. Rev Recent Clin Trials. 2007;2:77-84.

5. Zinman B, Hoogwerf BJ, Durán García S, et al. The effect of adding exenatide to a thiazolidinedione in suboptimally controlled type 2 diabetes: a randomized trial. Ann Intern Med. 2007;146: 477-485.

6. Buse JB, Bergenstal RM, Glass LC, et al. Use of twice-daily exenatide in basal insulin-treated patients with type 2 diabetes: a randomized, controlled trial. Ann Intern Med. 2011;154:103-112.

7. Heine RJ, Van Gaal LF, Johns D, et al. Exenatide versus insulin glargine in patients with suboptimally controlled type 2 diabetes: a randomized trial. Ann Intern Med. 2005;143:559-569.

8. Barnett AH, Burger J, Johns D, et al. Tolerability and efficacy of exenatide and titrated insulin glargine in adult patients with type 2 diabetes previously uncontrolled with metformin or a sulfonylurea: a multinational, randomized, open-label, two-period, crossover noninferiority trial. Clin Ther. 2007;29:2333-2348. 
9. Gailwitz E, Böhmer M, Segiet T, et al. Exenatide twice daily versus premixed insulin aspart 70/30 in metformin-treated patients with type 2 diabetes: a randomized 26-week study on glycemic control and hypoglycemia. Diabetes Care. 2011;34:604-606.

10. Derosa G, Maffioli P, Salvadeo SA, et al. Exenatide versus glibenclamide in patients with diabetes. Diabetes Technol Ther. 2010;12: 233-240.

11. Derosa G, Putignano P, Bossi AC, et al. Exenatide or glimepiride added to metformin on metabolic control and on insulin resistance in type 2 diabetic patients. Eur J Pharmacol. 2011;666:251-256.

12. Best JH, Hoogwerf BJ, Herman WH, et al. Risk of cardiovascular disease events in patients with type 2 diabetes prescribed the glucagonlike peptide 1 (GLP-1) receptor agonist exenatide twice daily or other glucose-lowering therapies: a retrospective analysis of the LifeLink database. Diabetes Care. 2011;34:90-95.

13. Garg R, Chen W, Pendergrass M. Acute pancreatitis in type 2 diabetes treated with exenatide or sitagliptin - a retrospective observational pharmacy claims analysis. Diabetes Care. 2010;33:2349-2354.

14. Wenten M, Gaebler JA, Hussein M, et al. Relative risk of acute pancreatitis in initiators of exenatide twice daily compared with other anti-diabetic medication: a follow-up study. Diabet Med. 2012;29: 1412-1418.

15. Romley JA, Goldman DP, Solomon M, McFadden D, Peters AL. Exenatide therapy and the risk of pancreatitis and pancreatic cancers in a privately insured population. Diabetes Technol Ther. 2012;14: 904-911.

16. DeYoung MB, MacConell L, Sarin V, Trautmann M, Herbert P. Encapsulation of exenatide in poly-(D,L-lactide-co-glycolide) microspheres produced an investigational long-acting once-weekly formulation for type 2 diabetes. Diabetes Technol Ther. 2011;13: 1145-1154.

17. Fineman M, Flanagan S, Taylor K, et al. Pharmacokinetics and pharmacodynamics of exenatide extended-release after single and multiple dosing. Clin Pharmacokinet. 2011;50:64-75.

18. Kim D, MacConell L, Zhang D, et al. Effects of once-weekly dosing of a long-acting release formulation of exenatide on glucose control and body weight in subjects with type 2 diabetes. Diabetes Care. 2007;30: 1487-1493.

19. Iwamoto K, Nasu R, Yamamura A, et al. Safety, tolerability, pharmacokinetics, and pharmacodynamics of exenatide once weekly in Japanese patients with type 2 diabetes. Endocr J. 2009;56:951-962.

20. Drucker DJ, Buse JB, Taylor K, et al. Exenatide once weekly versus twice daily for the treatment of type 2 diabetes: a randomised, openlabel, non-inferiority study. Lancet. 2008;372:1240-1250.

21. Buse JB, Druker DJ, Taylor KL, et al. DURATION-1: exenatide once weekly produces sustained glycemic control and weight loss over 52 weeks. Diabetes Care. 2010;33:1255-1261.

22. Taylor K, Gurney K, Han J, Pencek R, Walsh B, Trautmann M. Exenatide once weekly treatment maintained improvements in glycemic control and weight loss over 2 years. BMC Endocr Disord. 2011;11:9.

23. Blevins T, Pullman J, Malloy J, et al. DURATION-5: exenatide once weekly resulted in greater improvement in glycemic control compared with exenatide twice daily in patients with type 2 diabetes. J Clin Endocrinol Metab. 2011;96:1301-1310.

24. Ridge T, Moretto T, Macconell L, et al. Comparison of safety and tolerability with continuous (exenatide once weekly) or intermittent (exenatide twice daily) GLP-1 receptor agonism in patients with type 2 diabetes. Diabetes Obes Metab. 2012;14:1097-1103.

25. Pencek R, Blickensderfer A, Li Y, Brunell C, Chen S. Exenatide once weekly for the treatment of type 2 diabetes: effectiveness and tolerability in patient subpopulations. Int J Clin Pract. 2012;66: 1021-1032.

26. Bergenstal RM, Wysham C, MacConell L, et al. Efficacy and safety of exenatide once weekly versus sitagliptin or pioglitazone as an adjunct to metformin for treatment of type 2 diabetes (DURATION-2); a randomised trial. Lancet. 2010;376:431-439.
27. Wysham, Bergenstal R, Malloy J, et al. DURATION-2: efficacy and safety of switching from maximum daily sitagliptin or pioglitazone to once-weekly exenatide. Diabet Med. 2011;28(6):705-714.

28. Russell-Jones D, Cuddihy RM, Hanefeld M, et al. Efficacy and safety of exenatide once weekly versus metformin, pioglitazone, and sitagliptin used as monotherapy in drug naïve patients with type 2 diabetes (DURATION-4): a 26-week double-blind study. Diabetes Care. 2012;35(2):252-258.

29. Diamant M, Van Gaal L, Stranks S, Northup J, Cao D, Trautmann M. Once weekly exenatide compared with insulin glargine titrated to target in patients with type 2 diabetes (DURATION-3): an open-label randomised trial. Lancet. 2010;375:2234-2243.

30. Inagaki N, Atsumi Y, Oura T, Saito H, Imaoka T. Efficacy and safety profile of exenatide once weekly compared with insulin once daily in Japanese patients with type 2 diabetes treated with oral antidiabetes $\operatorname{drug}(\mathrm{s})$ : results from a 26-week, randomized, open-label, parallel-group, multicentre, noninferority study. Clin Ther. 2012;34:1892-1908.

31. Buse JB, Nauck M, Forst T, et al. Exenatide once weekly versus liraglutide once daily in patients with type 2 diabetes (DURATION-6): a randomised, open-label study. Lancet. 2012;pii:S0140-6736: 61267.

32. Scott DA, Boye KS, Timlin L, Clark JF, Best JH. A network metaanalysis to compare glycaemic control in patients with type 2 diabetes with exenatide once weekly or liraglutide once daily in comparison with insulin glargine, exenatide twice daily, or placebo. Diabetes Obes Metab. September 7, 2012. [Epub ahead of print.]

33. Bradley C. The Diabetes Treatment Satisfaction Questionnaire: DTSQ. In: Chur BC, editor. Handbook of Psychology and Diabetes: A Guide to Psychological Measurement in Diabetes Research and Practice. Chur, Switzerland: Harwood Academic Publishers; 1994.

34. Kolotkin RL, Crosby RD, Kosloski KD, Williams GR. Development of a brief measure to assess quality of life in obesity. Obes Res. 2001;9: $102-111$.

35. Best JH, Boye KE, Rubin RR, et al. Improved treatment satisfaction and weight-related quality of life with exenatide once weekly or twice daily. Diabet Med. 2009;26:722-728.

36. Dupuy HJ. The Psychological General Well-Being (PGWB) Index. In: Wenger NK, editor. Assessment of Quality of Life in Clinical Trials of Cardiovascular Therapies. New York, NY: LeJacq Publications; 1984.

37. Best JH, Rubin RR, Peyrot M, et al. Weight-related quality of life, health utility, psychological well-being, and satisfaction with exenatide once weekly compared with sitagliptin or pioglitazone after 26 weeks of treatment. Diabetes Care. 2011;34:314-319.

38. Fabunmi R, Nielsen LL, Quimbo R, et al. Patient characteristics, drug adherence patterns, and hypoglycemia costs for patients with type 2 diabetes mellitus newly initiated on exenatide or insulin glargine. Curr Med Res Opin. 2009;25:777-786.

39. Palmer AJ, Roze S, Valentine WJ, et al. The CORE diabetes model: projecting long-term clinical outcomes, costs and cost-effectiveness of interventions in diabetes mellitus (types 1 and 2) to support clinical and reimbursement decision-making. Curr Med Res Opin. 2004;Suppl 1: S5-S26.

40. DeFronzo RA, Ratner RE, Han J, et al. Effects of exenatide (exendin-4) on glycemic control and weight over 30 weeks in metformin-treated patients with type 2 diabetes. Diabetes Care. 2005;28:1092-1100.

41. Buse JB, Henry RR, Han J, et al. Effects of exenatide (exendin-4) on glycemic control over 30 weeks in sulfonylurea-treated patients with type 2 diabetes. Diabetes Care. 2004;27:2628-2635.

42. Kendal DM, Riddle MC, Rosenstock J, et al. Effects of exenatide (exendin-4) on glycaemic control over 30 weeks in patients with type 2 diabetes treated with metformin and a sulfonylurea. Diabetes Care. 2005;28:1083-1091.

43. Minshall ME, Oglesby AK, Wintle ME, Valentine WJ, Roze S, Palmer AJ. Estimating the long-term cost-effectiveness of exenatide in the United States: an adjunctive treatment for type 2 diabetes mellitus. Value Health. 2008;11:22-33. 
44. Guillermin A-L, Lloyd A, Best JH, DeYoung MB, Samyshkin Y, Gaebler JA. Long-term cost-consequence analysis of exenatide once weekly versus sitagliptin or pioglitazone for the treatment of type 2 diabetes patients in the United States. J Med Econ. 2012;15:654-663.

45. Beaudet A, Palmer JL, Timlin L, et al. Cost-utility of exenatide once weekly compared with insulin glargine in patients with type 2 diabetes in the UK. J Med Econ. 2011;14:357-366.

46. Samyshkin Y, Guillermin AL, Best JH, Brunell SC, Lloyd A. Long-term cost-utility analysis of exenatide once weekly versus insulin glargine for the treatment of type 2 diabetes patients in the US. J Med Econ. 2012;15 Suppl 2:6-13.

47. Valentine WJ, Palmer AJ, Lammert M, Langer J, Brändle M. Evaluating the long-term cost-effectiveness of liraglutide versus exenatide BID in patients with type 2 diabetes who fail to improve with oral antidiabetic agents. Clin Ther. 2011;33:1698-1712.

48. Eddy DM, Schelessinger L. Archimedes - a trial-validated model of diabetes. Diabetes Care. 2003;26:3093-3101.

49. Eddy DM, Schelessinger L. Validation of the Archimedes diabetes model. Diabetes Care. 2003;26:3102-3110.

50. Centers for Disease Control and Prevention National Center for Health Statistics. National Health and Nutrition Examination Survey Data. Hyattsville, MD: US Department of Health and Human Services, Centers for Disease Control and Prevention; 2010.

51. Peskin BR, Shcheprov AV, Boye KS, Bruce S, Maggs DG, Gaebler JA. Cardiovascular outcomes associated with a new once-weekly GLP-1 receptor agonist versus. traditional therapies for type 2 diabetes: a simulation analysis. Diabetes Obes Metab. 2011;13:921-927.
52. Gaebler JA, Soto-Campos G, Alperin P, et al. Health and economic outcomes for exenatide once weekly, insulin, and pioglitazone therapies in the treatment of type 2 diabetes: a simulation analysis. Vasc Health Risk Manag. 2012;8:255-264.

53. Home Safety MedWatch. The FDA Safety Information and Adverse Program Safety Information. Byetta (exenatide) injection. Available from: http://www.fda.gov/Safety/MedWatch/SafetyInformation/ ucm194556.htm. Accessed October 1, 2012.

54. Signorovitch JE, Wu EQ, Swallow E, Kantor E, Fan L, Gruenberger JB. Comparative efficacy of vildagliptin and sitagliptin in Japanese with type 2 diabetes mellitus: a matching-adjusted indirect comparison of randomized trials. Clin Drug Invest. 2011;31:665-674.

55. Berlie H, Hurrne KM, Pinelli NR. Glucagon-like peptide-1 receptor agonists as add-on therapy to basal insulin in patients with type 2 diabetes: a systematic review. Diabetes Metab Syndr Obes. 2012;5: $165-174$.

56. Ryan DH, Espeland MA, Foster GD, et al; Looking AHEAD Research Group. Look AHEAD study (Action for Health in Diabetes): design and methods for a clinical trial of weight loss for the prevention of cardiovascular disease in type 2 diabetes. Control Clin Trials. 2003;24: 610-628.

57. Jakicic JM, Egan CM, Fabricatore AN, et al. Four-year change in cardiovascular fitness and influence on glycemic control in adults with type 2 diabetes in a randomized trial. The Look AHEAD Trial. Diabetes Care. December 6, 2012. [Epub ahead of print.]

58. O'Riorhan M. Look AHEAD trial halted. Available from: http://www. medscape.com/viewarticle/772490. Accessed December 13, 2012.
Patient Preference and Adherence

\section{Publish your work in this journal}

Patient Preference and Adherence is an international, peer-reviewed, open access journal focusing on the growing importance of patient preference and adherence throughout the therapeutic continuum. Patient satisfaction, acceptability, quality of life, compliance, persistence and their role in developing new therapeutic modalities and compounds to

\section{Dovepress}

optimize clinical outcomes for existing disease states are major areas of interest. This journal has been accepted for indexing on PubMed Central. The manuscript management system is completely online and includes a very quick and fair peer-review system. Visit http://www.dovepress.com/ testimonials.php to read real quotes from published authors. 